\title{
Protée
}

\section{Du sens}

\section{Prolongements théoriques autour de la perception et de la modalisation}

\section{Cécilia W. Francis}

Volume 34, numéro 1, printemps 2006

Fortune et actualité de $\mathrm{Du}$ sens

URI : https://id.erudit.org/iderudit/013308ar

DOI : https://doi.org/10.7202/013308ar

Aller au sommaire du numéro

\section{Éditeur(s)}

Département des arts et lettres - Université du Québec à Chicoutimi

ISSN

0300-3523 (imprimé)

1708-2307 (numérique)

Découvrir la revue

Citer cet article

Francis, C. W. (2006). Du sens : prolongements théoriques autour de la perception et de la modalisation. Protée, 34(1), 33-45.

https://doi.org/10.7202/013308ar

\section{Résumé de l'article}

La présente réflexion met en lumière la portée prophétique de $\mathrm{Du}$ sens d'A. J. Greimas. L'auteure s'attarde sur les notions-clés d'engendrement du sens discursif et de conditions de la production des récits en circonscrivant une filiation entre ces concepts élaborés dans l'ouvrage de 1970 et leurs prolongements et affinements inaugurés par la sémiotique des passions. On fait état ici de deux sphères principales de théorisation ayant leur ancrage dans l'ouvrage $D u$ sens, à savoir le rôle de la perception dans la mise au jour et le façonnement discursif de la signification ainsi que le corrélat syntaxique de la perception, incarné notamment par les modalités, des procès de modalisation et d'aspectualisation, porteurs de proprioceptivité au plan du discours. Faisant valoir la contribution du texte fondateur de Greimas aux préoccupations contemporaines de la sémiotique, l'article contribue à dédramatiser l'image de rupture qui tend à persister entre les deux visages de la sémiotique européenne, discontinue et continue.
Ce document est protégé par la loi sur le droit d'auteur. L'utilisation des services d’Érudit (y compris la reproduction) est assujettie à sa politique d'utilisation que vous pouvez consulter en ligne.

https://apropos.erudit.org/fr/usagers/politique-dutilisation/ 


\title{
DU SENS: PROLONGEMENTS THÉORIQUES AUTOUR DE LA PERCEPTION ET DE LA MODALISATION
}

\author{
CÉCILIA W. FranCIS
}

Évaluer la portée prophétique de Du sens à la lumière de l'expansion de la théorie sémiotique vers une sémiotique tensive, qui interroge le sens en tant que procès continu, nécessite un effort de contextualisation et d'articulation d'un certain nombre de filiations notionnelles en attente d'éclairage. Un tel travail de mise au jour de points de présupposition et d'emboitement entre la théorie des passions et son héritage épistémologique s'impose, étant donné que la première, loin de constituer un simple ajout à l'édifice de base, complète le portrait de la sémiotique cognitive et demeure donc redevable aux fondements de la théorie de l'action l'ayant nourrie en son amont. Du sens $(1970)^{1}$ représente une assise appropriée pour cet exercice de consolidation: l'ouvrage constitue un jalon essentiel de théorisation coextensif à la sémiotique structurale destiné à l'élucidation des procédures de transcodage inhérentes au parcours génératif, soit la conversion de la sémantique profonde, établie sur des unités de sens discrètes (discontinues), en structures sémio-narratives d'univers anthropomorphes et en parcours complexes au niveau du discours. Si la théorie standard ainsi esquissée, qui alimente le premier tome du Dictionnaire raisonné de la théorie du langage (1979), se consacre à formuler les conditions de la semiosis engageant la narrativité (ancrée à même le /faire/) et l'armature logiciste sous-tendant des artefacts culturels, tant artistiques qu'utilitaires, la sémiotique des passions, dite tensive, atteignant sa formalisation au début des années 1990, a pour socle les préconditions phénoménologiques de l'apparition du sens et vise d'autres zones de signification impliquant notamment l'affect, la proprioception et le corps. Il s'agit d'une voix sémiotique parallèle, moins imprégnée de l'esprit cartésien que vouée à saisir l'évolution des grandeurs labiles en tant que problématiques associées à l'/être/ (Broden, 1995: 233). L'intuition d'A.J. Greimas est à la base de cet élargissement heuristique de la théorie, où se manifeste clairement «sa volonté, entre autres, d'articuler l'intelligible et le sensible sans perdre de vue la valeur ou l'axiologie» (Ouellet, 1997: 8). Circonscrivant l'apport du texte fondateur de Greimas aux préoccupations contemporaines de la sémiotique, le présent article a pour objectif d'atténuer l'image de rupture qui tend à persister entre les deux visages de la sémiotique greimassienne, discontinue et continue. À cette fin, on propose de faire 
état de deux faisceaux majeurs de théorisation propres à la sémiotique des passions qui ont tous deux leur ancrage conceptuel dans Du sens. Il est question de mettre en lumière et d'examiner les liens de recoupement et d'affinement théoriques qui touchent au rôle de la perception dans l'engendrement de la signification ainsi qu'à la problématique des modalités, des procès de modalisation et d'aspectualisation, corrélats phoriques et syntaxiques de la perception, porteurs de la proprioceptivité sur le plan du discours.

\section{AutOUR DE LA SAISIE PERCEPTIVE}

Dans l'introduction éponyme de l'ouvrage $D u$ sens, Greimas propose une réflexion en vue de l'établissement d'une sémiotique formelle visant à la démarquer de la sémantique interprétative. Il offre deux sphères principales d'élaboration épistémologique. D'une part, il signale l'importance d'isoler un minimum de concepts qui permettent au sémioticien d'apprécier, lorsqu'il est question d'analyse de significations, l'adéquation des modèles proposés. D'où le souci de construire un «langage artificiel adéquat» (DS: 14), un métalangage, grâce auquel s'établit un système d'équivalences «des rapports entre les modèles de description et la structure élémentaire de la signification" (DS: 14). D'autre part, sachant que le langage, tout comme le discours, n'est jamais dénotatif, ni monoplan, il s'efforce de concevoir «les formes multiples de la présence du sens et les modes de son existence» (DS: 17). Car le sens, «pour se manifester, peut prendre tantôt la forme du système, tantôt celle du procès" (DS: 16); c'est dire qu'un «faire peut être à la fois transcrit comme un algorithme processuel et transcodé comme un savoir-faire systématique et virtuel» (DS: 16). Une telle approche lui permet d'assurer une «équivalence entre les axiologies d'ordre systématique et les idéologies qui sont des représentations - récurrentes - des procès de transformation" (DS: 16). En somme, Greimas élabore ainsi un des théorèmes clés de sa sémiotique formelle, à savoir l'idée que «[1]e sens, en tant que forme $d u$ sens, peut se définir alors comme la possibilité de transformation du sens" (DS: 15; l'auteur souligne). Son projet se résume, en effet, en une description des "parcours des transpositions et transformations de contenus» (DS: 17).

De manière concomitante, le texte inaugural de $D u$ sens revient sur un axiome rudimentaire touchant au jeu natif $d u$ sens - ce qui ne cessera de hanter le maître tout au long de sa carrière, au point de s'imposer en tant que postulat charnière de la sémiotique des passions. Dans son introduction, passant en revue les acquis linguistiques de "nos anciens" (DS: 8) (dont Saussure et Bloomfield), Greimas reconnaît leur mérite d'«étudier les conditions de la manifestation du sens" (DS: 8) à partir du plan du signifiant (sonore ou graphique). Or, il signale que l'entreprise de ces linguistes n'a pas donné les résultats escomptés en ce qui concerne les morphèmes, puisqu'on visait à étudier, à l'aide de procédés formels, le passage d'un niveau à l'autre, «d'un en-deçà du sens à la distribution des significations»(DS: 9). Greimas précise que ces linguistes nous ont tout de même légué le concept de sens négatif, "cette possibilité de dire que "pas" n'est pas "bas", qu'il y a entre les deux un écart de sens» (DS: 9; l'auteur souligne). Il est à noter que la notion d'écart différentiel relève directement de la perception, de la compétence du sujet percevant à saisir les moindres variables de sens ayant leur corollaire du côté du signifiant. Poursuivant sa réflexion sur «les conditions premières de la saisie du sens» (DS: 10), Greimas évoque la métaphore saussurienne d'une toile d'araignée placée devant un écran de fumée pour parler de notre perception du sens: les écarts différentiels en question ne sont «que des conséquences de la saisie [perceptive] des discontinuités» (DS: 9). Si les écarts permettent d'établir une relation de différence entre les aspects comparables des choses, c'est la saisie perceptive qui revient à la charge, étant donné que «cette saisie est logiquement antérieure à l'écart reconnu " (DS: 10). Le maitre pousse plus loin ses observations en stipulant que

[...] la substance du signifiant n'est qu'un prétexte permettant la saisie du sens, qu'elle est «informée» par ladite saisie, que la 
forme du signifiant, c'est-à-dire l'ensemble des écarts, résulte, comme une articulation, des opérations de la saisie. (DS: 10)

Fait significatif, on voit s'esquisser, à ce stade de la sémiotique encore fortement investi d'une visée structuraliste, un retour sur «un en-deçà du sens» (DS: 9), sur le rôle primordial du corps et de la perception dans l'émergence de la signification. Du point de vue épistémologique, bien que Greimas ne soit pas encore en mesure d'identifier formellement l'effet de ces préconditions de la signification sur un engendrement logique du sens fondé à partir d'un système de sèmes binaires, il réitère ici son allégeance à la phénoménologie de Maurice Merleau-Ponty.

Rappelons que le postulat évoqué l'avait amené à poser, dès Sémantique structurale, que la perception est "le lieu non linguistique où se situe l'appréhension de la signification» (Greimas, 1966: 8) et que celle-ci se trouve par conséquent infléchie par la sphère non catégorielle des sensations et des affects. L'énoncé d'un tel postulat tient, en effet, en germe un principe fondateur de la sémiotique des passions, à savoir que «[c]'est par la médiation du corps percevant que le monde se transforme en sens» (Greimas et Fontanille, 1991: 12).

La question du corps et de ses capacités sensorielles, quoique fondamentale pour Greimas, devait rester en friche encore une quinzaine d'années. Précédant l'intégration d'une théorisation sur le rôle du sensible dans le façonnement du sens à l'intérieur de l'économie théorique de la sémiotique, cette période fut, pour l'École de Paris, caractérisée tantôt par des reproches, provenant notamment de la critique post-structurale et de l'analyse discursive, tantôt par des digressions théoriques autour de la notion de générativité menées à l'intérieur même du paradigme sémiotique (à savoir, la théorie des catastrophes, la sémanalyse, la sémiotique subjectale). Dans la foulée des interrogations sur l'universalité des structures sémiotiques tant paradigmatiques (le modèle constitutionnel) que syntagmatiques (le modèle transformationnel), on a souligné que ces dernières étaient en défaut lorsqu'il s'agissait d'articuler les notions d'hétérogène, de gradation, d'expansion, d'intensité ou de rythme qui, tout en étant impératives pour la signification, concernent de près les procédures aspectuelles de pathémisation. Paul Ricœur, entre autres, a soulevé que l'analyse effectuée par Greimas de «Deux amis» de Maupassant, où sont privilégiées les récurrences et les permanences, demeure à bien des égards incomplète. D'après Ricœur, l'étude aurait gagné en précision à être doublée de facteurs processuels, à prendre en compte le "développement d'une amitié», «une amitié en croissance», «l'approfondissement d'un pacte d'amitié» (1985: 801-809). Cela aurait obligé à articuler autrement le dynamisme inhérent aux structures narratives. Car le déploiement d'une structure ne se ramène pas seulement à l'occupation d'un espace et à la subsistance dans une durée, soit la répétition ou la multiplication, la conservation et la différenciation au nom d'une stabilité interne. Comme le précise Claude Zilberberg, il y est plutôt prise en charge des catégories à la fois formelles et existentielles (le temps, le mode, l'aspect, le nombre, le rythme, le tempo, le pâtir...), par lesquelles «le sujet tensif change, pense changer le lieu en place, le survenir en devenir, la déréliction en consolation" (1992a: 14). Cette dynamique caractérise à la lettre les parcours identitaires d'un sujet passionnel. Tous deux ayant plaidé en faveur d'une meilleure intégration des phénomènes d'opérativité (le procès) à la structure (le système) (Ricœur, 1993: 46-47), l'herméneute tout comme le sémioticien ont fait écho à la revendication de concepts dynamiques pour les sciences du langage. Or, sur ce plan, la sémiotique greimassienne n'était pas en reste, puisqu'elle repose traditionnellement sur l'idée que le sens n'est saisissable que dans ses transformations. Cette conception dynamique la destinait à devenir une sémiotique narrative. Mais la stricte saisie cognitive du dynamisme, au cours des années 1970 et au début des années 1980, a eu pour résultat d'orienter la méthodologie vers une prise en compte des unités contraires et disjointes. L'adoption d'une catégorisation binaire, d'après l'exploitation magistrale effectuée par Claude Lévi-Strauss dans son 
analyse des mythes, a entrainé une méconnaissance des formulations aptes à articuler la propriété extensionnelle du sens (Zilberberg, 1992b: 85). Seraitce pour cette raison que, malgré les tentatives ponctuelles de dépasser le binarisme logique, observées à titre d'indice dans la version scalaire que Greimas propose du carré sémiotique pour rendre compte de l'acte épistémique ${ }^{2}$, la théorie a buté sur la description des régimes discursifs du continu? Toujours est-il qu'il fallait attendre l'élargissement et la refondation de l'édifice sémiotique dans l'optique des concepts fluents de la signification avant de reconnaître la portée heuristique de ce que Greimas nomme, dans Du sens, «un en-deçà du sens», lequel a permis d'affiner la notion de transformation.

On pose un jalon important dans l'intégration de la saisie perceptive en commençant à s'intéresser à la scénographie discursive sous-tendant la figurativisation. Une perception en acte, ce genre d'archi-événement accompagné de ce que Greimas baptise l'esthesis, représente l'endroit par excellence de la dissémination d'une perception originaire, qui ne se laisse pas saisir dans un réseau binaire d'axes sémantiques. Notons à cet effet que la discursivisation de l'esthesis implique un débrayage actoriel, soit la mise en place d'un sujet qui, par le truchement de sa modalisation perceptive, participe à la construction de l'univers référentiel. Si les travaux de Claude Zilberberg $(1992,1993)$ sur le tempo et ceux de Pierre Ouellet $(1992,2000)$ sur le noème ont montré que le sujet perceptif crée son monde par sa disposition sensori-motrice, l'espace-temps dans lequel il évolue, Greimas a surtout exploité, dans De l'imperfection (1987), la composante proprioceptive de ce rapport en vue de consolider la dimension esthétique du discours ${ }^{3}$. Il vérifie ses intuitions à partir des exemples littéraires, en situant l'émotion esthétique au moment du re-sentir chez le sujet de la scission première, d'une "tensivité phorique» indifférenciée (Greimas et Fontanille, 1991: 30). Le chercheur insiste ainsi sur la composante sensorielle de la perception, laquelle fonde pour le signifiant, pour l'objet perçu, son signifié et établit, à partir de la transformation fondamentale de la relation entre le sujet et l'objet, un nouvel «état de choses" (Greimas, 1987: 76). La convocation du sujet par la forme immanente des figures du monde sensible coïncide, en ce cas, avec la révélation du sens (Landowski, 1997: 234). Fort d'une lecture de textes littéraires contemporains (Calvino, Tournier, Junichiro, Rilke, Cortázar), plus poétique que fondée sur des contraintes méthodologiques de la sémiotique narrative, Greimas commence à creuser plus profondément ses premières hypothèses, émises dans $D u$ sens, et illustre la mise en forme de la saisie du sens par la saisie perceptive (DS: 10).

\section{PHÉNOMÉNOLOGIE ET PRÉCONDITIONS \\ DE LA SIGNIFICATION \\ Cependant, il fallait au préalable que} l'épistémologie interne de la théorie "cess[e] de voir son modèle exclusif dans la linguistique" (Klinkenberg, 2002: 548). Rappelons à cet égard que, si plusieurs principes phénoménologiques, combinés aux données structurales issues de la linguistique et de l'anthropologie, permettent à Greimas de consolider l'axiomatique d'une approche logarithmique du sens - à savoir, la forme du sens est informée par la saisie perceptive (dite opération) située antérieurement à la différence, à l' «écart» (DS: 9-10) -, une reprise plus nuancée de la pensée de Merleau-Ponty contribue à l'élaboration d'une théorie sémiotique des passions et de la perception. Poursuivant dans le sillage d'une phénoménologie corporelle, A. J. Greimas avec Jacques Fontanille proposent, dans Sémiotique des passions (1991), une vingtaine d'années après la publication de $D u$ sens, un scénario imaginaire pour rendre compte du rôle déterminant de l'acte perceptif dans le «jaillissement du sens» (DS: 10). Ils postulent, sous-jacent à la signification cognitive, un simulacre phorique régi par une pulsion archaïque traversée de modulations d'attraction et de répulsion, qui se font et se défont, et enrichissent ainsi le noyau structuraliste de la sémiotique d'une conception énergétique du monde calquée sur l'expérience perceptive. Rejoignant les nouvelles tendances continuistes propres à l'épistémologie des sciences 
dans son ensemble ${ }^{4}$, cette option énergétique permet aux chercheurs de situer l'apport corporel à l'éclosion de la signification dans un espace de tensivité phorique résultant d'une polarisation, d'une scission qui affecte une "masse thymique» fondatrice. Expliciter ce genre de tensivité originelle, c'est reprendre, à l'instar des sciences de la nature, la problématique philosophique de l'«un» et du "multiple»: le monde est-il un, débordant sa plénitude, une structure du mixte prête à éclater, ou un mélange chaotique tendant vers l'unité? D'après Greimas et Fontanille (1991: 19), la tensivité énergétique dérive précisément de cet éclatement de l'un, de la confrontation du "hasard " à la "nécessité", soit de l'introduction d'une «fracture" protensive au devoir-être de l'unicité, accident dit épistémologique qui rend pensable les premières esquisses de la syntaxe $^{5}$, tout comme les préfigurations du sujet et de l'objet conditionnant l'avènement du sens. En vertu d'une reformulation de l'acte perceptif, les auteurs installent ainsi au cour de la relation sujet-objet une zone de médiation sensible. D'où l'idée, évoquée dans $D u$ sens, du corps sentant qui travaille l'espace potentiel de la signification, réclamant de ce fait une représentabilité propre. L'implication

phénoménologique permet de concevoir le sujet à partir de son ek-sistence, de son incarnation, et de penser ensemble ses appartenances au monde, à l'autre et au langage, non sur le mode de l'extériorité, mais dans un rapport d'inclusion réciproque. Cette lecture du sujet s'envisage avant tout dans sa relation constitutive à un dehors qui forcément l'altère, comme dans l'instance d'expériences passionnelles (Collot, 1997: 32-33). Découle de cette semiosis proprioceptive, fondatrice en ce qui concerne la dimension thymique ${ }^{6}$, une équivalence entre les "états de choses» du monde et les "états d'âme» du sujet (Greimas et Fontanille, 1991: 13-14). Cette condition première garantit à la théorie un espace formel d'existence sémiotique, dont émergent non seulement la signification, mais encore la possibilité de valorisations potentielles d'ordre affectif ou passionnel. C'est dire que la mise en commun de la subjectalité de l'objet et de l'objectalité du sujet permet d'homogénéiser l'existence sémiotique. Ainsi, la signification ne relève pas seulement de la sphère logique des concepts, mais aussi, et surtout, du substrat proprioceptif qui la fonde. Grâce donc à la mise de l'avant de l'existence sémiotique, l'acception initialement cognitive et catégorielle du signe se trouve élargie, puisque les «différenciations continues" (Hénault, 1992b: 191) d'ordre protensif, régissant la signification en amont de son articulation, conditionnent le déploiement de celle-ci, tant sur le plan du contenu que sur le plan de l'expression. Il ne s'agit pas de nier la différence linguistique, à savoir l'«écart» catégoriel. On observe, en revanche, que cette différence, indispensable à l'apparition du sens, se trouve infiniment nuancée à l'aide des grandeurs proprioceptives susceptibles de traduire le sensible sous forme de degrés, de rythme, d'intensité - autant de morphologies graduelles qui concourent à poser la conquête du sens en termes qualitatifs de "diffusion" (Zilberberg, 1992a: 2).

\section{DES VALEURS FLUENTES À LA MODALISATION}

Quelle est l'incidence de cette réarticulation de l'engendrement du sens sur la mise en forme de la proprioception sur le plan du discours? Principe de base de la sémiotique greimassienne, qu'elle soit discontinue ou continue, ce sont les modalités et les procès de modalisation ensemble, avec leurs contreparties aspectuelles, qui sont porteurs de la proprioceptivité archaïque et génératrice du sens, apparaissant souvent sur le plan textuel sous forme de scénarios passionnels. À cet effet, force est de préciser que dans $D u$ sens, bien que Greimas se limite à mettre en place les unités élémentaires d'une grammaire superficielle, certains concepts élaborés soutiennent déjà en essence la complexification que connaîtra le dispositif modal. Dans le chapitre en question, "Éléments d'une grammaire narrative», il examine principalement le fonctionnement de "l'unité syntaxique typique qu'est la performance» (DS: 182). Partant du principe que la narration est un «processus créateur de valeurs» (DS: 178), il propose de retenir 
deux séries de performances: «a) les performances destinées à l'acquisition et à la transmission des valeurs modales et b) les performances caractérisées par l'acquisition et le transfert des valeurs objectives" (DS: 179). Les premières instituent les sujets comme opérateurs, alors que les secondes effectuent les opérations (les premières créent des virtualités, les secondes les actualisent). Le chercheur offre ensuite une première hiérarchie de valeurs modales capable d'orienter le parcours syntagmatique en vue de la performance, soit la suite modale: vouloir $\rightarrow$ savoir $\rightarrow$ pouvoir $\Rightarrow$ faire (DS: 179). Quoique Greimas situe la mise en marche de l'unité syntaxique que constitue la performance narrative à même la modalité du vouloir, ses élaborations théoriques se consacrent principalement aux modalités de savoir et de pouvoir. Ces dernières déterminent le faire performatif éventuel de deux manières différentes: comme un faire soit issu du savoir (le savoir-faire - ruse, tromperie), soit se fondant uniquement sur le pouvoir (le pouvoir-faire énergie, puissance) (DS: 175). En ce qui a trait au vouloir, alors qu'il signale que cette modalité a pour fonction d'instaurer le sujet comme virtualité d'un faire, il s'en tient à une explication pour le moins énigmatique.

Nous ne pouvons pas nous étendre ici sur l'origine du premier actant-opérateur qui déclenche le parcours syntaxique: cela nous entraînerait à examiner de près l'unité particulière qu'est le contrat instituant le sujet du désir par l'attribution de la modalité du vouloir, actualisation probable d'un "faire-vouloir» $d u$ destinateur originel. (DS: 179; l'auteur souligne)

La relégation du vouloir instaurateur de la performance, impliquant «le sujet du désir», vers un moment ultérieur de consolidation théorique n'est toutefois, à notre sens, que partielle. Car, dans une partie préliminaire du chapitre (DS: 167), le chercheur éclaire certains attributs de ce fonctionnement modal, qu'on verra repris et grandement enrichi dans sa théorie des passions. La réflexion la plus essentielle dans $D u$ sens, relativement à cette problématique, se rapporte à l'établissement d'un lien explicite entre la modalité du vouloir, définie en tant que «classème anthropomorphe» (DS: 168), et l'instauration de l'actant en «sujet [...] opérateur éventuel du faire» (DS: 168-169). C'est dire que

[...] l'introduction du classème "vouloir» est autre chose qu'une surdétermination du prédicat, qu'elle nécessite la construction de deux énoncés distincts dont le premier est un énoncé modal et le second un énoncé descriptif qui, hypotaxique par rapport au premier, lui sert d'Actant-Objet. (DS : 169)

Constituée en énoncé modal, cette relation peut s'interpréter comme «le désir de réalisation" (DS: 169) d'une action, l'actant-objet de l'énoncé modal pouvant être converti à tout instant en n'importe quel énoncé descriptif.

En poursuivant son argumentation, Greimas nuance ce qu'il dénomme l'ordre du faire (narratif) par la prise en compte de deux autres types d'énoncés descriptifs (reliés à la modalisation du sujet par le vouloir), qui relèvent «tantôt de l'ordre de l'avoir, tantôt de l'ordre de l'être» (DS: 170). Notons que Greimas cible ainsi, avant la lettre, l'axe modal de l'émotivité et des articulations du passionnel sur le plan du discours ${ }^{7}$. On observe que les deux nouveaux types d'énoncés descriptifs, qui s'ajoutent au premier, servent à décrire une relation d'attribution entre un sujet et un objet sémantique, soit la nature externe (Pierre veut une pomme) ou interne (Pierre veut être bon) des objets désirés et attribuables au sujet. En réunissant les fonctions de ces deux énoncés, on peut dire que le désir de possession (avoir) institue l'objet d'une possession virtuelle, la pomme, comme une valeur externe par rapport au sujet du désir, tandis que la bonté, soit le désir d'un état (être), s'avère une valeur interne au sujet. Greimas résume son propos en stipulant que l'introduction dans la grammaire superficielle de la modalité du vouloir permet une construction modale à deux actants, à savoir le sujet et l'objet; cet «axe du désir» (DS: 170) autorisant à son tour de les interpréter comme un «virtuel sujet performateur et un objet institué en valeur» (DS: 171, l'auteur souligne). Il va de soi que ce genre de relation syntagmatique peut s'orienter en fonction d'un programme d'action ${ }^{8}$. Mais, en mettant en lumière les 
valeurs attributives dans la possession d'objets valeurs (pouvant relever de l'ordre affectif), Greimas différencie deux types de valeurs, soit externes au sujet (hypotaxiques), soit internes à lui (hyponomiques), et, ce faisant, esquisse un critère formel permettant de distinguer deux ordres de valeurs dites soit objectives, soit subjectives (DS: 171). Le chercheur termine en soulignant que celles-ci sont «d'une importance capitale pour la compréhension de la structure narrative" (DS: 171), ce qui se confirmera à la lumière d'une sémiotique des passions.

L'articulation, dès $D u$ sens, des énoncés syntagmatiques hyponomiques, internes et subjectifs, qualifiant le rapport se nouant entre sujet et objet, autorise à isoler une dynamique modale rudimentaire et à y circonscrire les linéaments d'une relation existentielle, sorte de chaînon essentiel qui marque et assure le relais entre la sémiotique de l'action et la sémiotique des passions. Dans leur démarche, Greimas et Fontanille (1991) raffinent et nuancent, en effet, les enjeux modaux de l'interactantialité qui remontent à l'ouvrage de 1970. Amendant le dualisme catégoriel de la narrativité, les chercheurs signalent que le fonctionnement des passions exige de repenser la perspective narrative de l'action, où le /faire faire/ du Destinateur institue pour le sujet l'objet de valeur. En revanche, c'est à un presque-sujet pré-catégoriel, investi d'un objet digne de valorisation, de spécifier la nature protensive de ce rapport ${ }^{9}$ : au moment de la scission de l'un en double phorique émerge une fiducie ayant pour effet de canaliser les oscillations de l'attraction et de la répulsion. Sans établir une jonction véritable, cette fiducie n'en représente pas moins une projection énergétique qui modalise l'interface d'implication enclenchée. Isomorphe à un méta-croire, traduisible à juste titre en termes de «pressentiment» (Greimas et Fontanille, 1991: 27) quant à l'objet informe identifié à une valence, la fiducie signale une première modulation au sein de la tensivité amorphe. Sous cet angle, la visée prédicative d'ordre cognitif se trouve recatégorisée en termes d'effet de visée intensif résultant d'une orientation fiduciaire. Car, dans l'espace de la phorie, le sujet et l'objet, participant d'un premier moment d'indifférenciation protothymique, se comportent comme des effets de second degré, soit l'effet source et l'effet but de la masse thymique (ibid.: 46). C'est à ce niveau archaïque, pré-passionnel, que se situe l'empathie de Kant, laquelle présuppose la possibilité même de l'univers pathique. Dans cet «en-deçà» du sens, on capte le repli des passions sur elles-mêmes, "un "creux intérieur producteur" réunissant l'ensemble de conditions de possibilité de la vie passionnelle» (Parret, 1986: 80) ${ }^{10}$. Éternel retour de la passion sur elle-même, l'empathie s'instaure en tant que compétence passionnelle, à partir de laquelle on peut concevoir le fonctionnement de l'affectivité dans la vie et sur le plan du discours. Le sujet protensif renvoie en ce sens à un proto-actant issu de l'ébranlement originaire qui, capable d'intérioriser en vertu de la fiducie tous les attributs tensifs d'un déploiement passionnel, serait susceptible de les adopter, indépendamment du rôle actantiel effectif qui lui est réservé, sur la dimension pragmatique ou cognitive du discours. Expulsant ainsi toute référence au Destinateur, le régime prototensif fait intervenir des composantes variables de divergence et de réciprocité, inhérentes à une unicité énergétique, ce qui permet de poser avec une finesse accrue l'interrelation existentielle.

\section{DE LA MODALISATION AUX SIMULACRES TENSIFS}

Une des ouvertures indéniables de la recherche de Greimas et de Fontanille, par rapport à $D u$ sens, consiste à dévoiler l'autonomie de la syntaxe passionnelle au regard du schéma narratif canonique: l'organisation modale des séquences pathémiques constitue un dispositif autosuffisant dans la mesure où ce dernier se veut autre chose que la simple condition de la performance. Élaborer une sémiotique des passions, c'est donc prendre parti pour une dimension narrative des discours "qui ne se rédui[t] pas à une sorte de logique de l'action [ni] à une conception du sujet qui serait entièrement déterminé par son faire [...]» (Greimas et Fontanille, 1991: 99) ${ }^{11}$. La pathémisation de ce dernier relève plutôt d'un 
agencement modal de l'être, où un excès thymique vient régir son enchaînement processuel. Les modalisations qui ont fait l'objet d'une élaboration considérable à partir de $\mathrm{Du}$ sens ont donc constitué la porte d'entrée des développements dans le domaine du passionnel; elles sont ainsi tout à fait justiciables d'une teneur pathémique, grâce à la convocation énonciative de la base phorique présupposante, capable de leur assurer un dynamisme autre que catégoriel ${ }^{12}$.

Dans l'optique d'une théorie des passions, le processus de la modalisation, tout en s'appuyant sur les principes de la théorie standard, s'en distingue: en suivant la chaîne du parcours génératif, à la suite d'une première catégorisation, les énergies du palier ontique se transposent au niveau sémio-narratif dans un rapport de jonction où sujet et objet prennent part à une relation dite existentielle. Le sujet détermine l'axiologie de ce rapport du fait que ses réactions proprioceptives décident du statut de l'objet visé: en investissant celui-ci de valeur thymique (euphorie/ dysphorie), le sujet adopte lui-même la valeur censée investir l'énoncé tout entier, soit un Vouloir instaurateur. Il suffit donc qu'il soit doté d'un vouloirêtre pour que "la valeur $d u$ sujet se change en valeur pour le sujet au sens axiologique de ce terme» (Greimas, 1983: 23; l'auteur souligne). Cette prise en charge affective alimente en retour la structuration modale diversement articulée ${ }^{13}$. À titre d'illustrations, on observe ce genre de processus modal dans Bonheur d'occasion, notamment, chez Rose-Anna Lacasse, dont la honte face à sa condition familiale cède à la force d'un Vouloir, d'un désir pourtant jamais pleinement réalisé, de sortir les siens de la pauvreté. Chez Alexandre Chenevert, protagoniste du roman éponyme, le Vouloir instaurateur s'incarne dans un désir vif et douloureux de communication humaine, mais qui ne se solde que par des échecs et des frustrations répétés. De tels exemples réconfortent les acquis dans le domaine des modalités, depuis les fonctions initiales du sujet proppien jusqu'à la reconnaissance d'une syntaxe modale autonome (Bertrand, 1984: 15-17) ${ }^{14}$ libérée d'attaches actantielles; de plus, ils confirment que la jonction est bien le lieu d'«un surplus de sens» (Greimas, 1983: 95), attribuable à la modalisation présupposante. Forte de ses surdéterminations intermodales, mettant en jeu les concaténations reposant sur les modalités canoniques /vouloir/, /pouvoir/, /savoir/, /devoir/ et /croire/, la jonction est à même de nuancer considérablement la catégorisation axiologique. Il s'en dégage ainsi des modes d'existence du sujet narratif, saisis en termes d'identités modales transitoires. Ces dernières concourent à la mise en place d'une disposition passionnelle, propre à spécifier la position de l'objet de valeur (Brandt, 1986: 164) et, partant, à circonscrire un domaine actantiel de l'affect.

Par ailleurs, loin de se limiter au rapport sujetobjet, la relation existentielle peut innerver soit un programme narratif, soit le rapport entre sujets. Une telle interaction soulignant la dimension thymique du texte se résume par une modification réciproque et continue des "conflits et tensions» (Latella, 1986: 215) ${ }^{15}$ participant d'une même unicité fondatrice. Peu importe donc ses investissements figuratifs, le rapport sujet-objet présuppose une inter-définition: que le sujet d'état soit à la fois tendu vers l'objet et affecté par lui, l'objet, on l'a vu, n'acquiert de valeur à moins d'une valorisation d'ordre proprioceptif. Ainsi, le sujet de la passion, même responsable d'un faire, serait-il toujours modalisé selon l'être. La passion concerne en ce sens un sujet de second rang, cet être de faire, saisi non pas en tant que sujet agissant, mais comme sujet actif. Ce terme fait écho aux notions merleau-pontiennes d' «être en situation» et d'«investissement» (Merleau-Ponty, 1976: 488) qui permettent de capter le sujet de la passion non pas seulement en vertu d'une action causale, mais aussi et surtout en fonction d'une attitude réceptive. En plus de ses charges thymiques définies en termes de catégorie modale (/vouloir/, / pouvoir/... ) dont fait état $D u$ sens, le sujet passionné est affecté par les modes d'existence, par les jeux de la présence ou de l'absence de la protensivité.

C'est au chapitre de la mise en discours de la masse thymique implicite que l'on observe l'extension 
la plus importante en ce qui concerne la compétence modale du sujet élaborée initialement par la théorie standard. Pour qu'une suite modale relève bien du passionnel, une corrélation entre des modes d'existence narratifs d'un programme (virtualisation, actualisation, potentialisation, réalisation) et ceux de la jonction reliant un sujet avec son objet (nonconjonction, disjonction, non-disjonction, conjonction) doit être prise en charge par la protensivité. Autrement dit, l'équipement modal de l'actant (la phorie) nécessite qu'il soit doublé d'une jonction fictive (la tensivité) - tout aussi indispensable que la jonction effective -, à savoir les «simulacres existentiels» qui tiennent d'un «imaginaire» modal (Greimas et Fontanille, 1991: 59) ${ }^{16}$. Fondé sur un univers cognitif de référence, grâce auquel le sujet "peut embrayer ce qui se passe en lui [...] sous forme de pensées ou sous forme de sentiments» (Pozzato, 1991: 16), le simulacre et son emploi dans le domaine des passions demeurent une composante bien éprouvée. Alors que les suites prédicatives saisies en dehors de la configuration passionnelle renvoient à une organisation catégorielle incapable de rendre compte de transformations intermodales ${ }^{17}$, le simulacre existentiel, ce paraître de l'être tombant sous la gouverne d'une modalité centrale, investit le parcours passionnel d'une force régissante, lui attribue une cohésion syntaxique, là où il s'agit

d'enchaînements modaux hétérogènes, voire incompatibles. Car le simulacre est soutenu par la protensivité présupposante en raison de sa convocation par la praxis énonciative: sur le plan discursif, il revêt une forme aspectuelle, chargée de l'enchaînement fluide des prédicats. Ainsi pourrait-on aborder la schématisation discursive des passions dans l'optique d'un simulacre dominant, d'une image but réarticulée par Greimas et Fontanille en termes de «trajectoire existentielle». Ce genre de fantasme protensif permet d'expliquer qu'un avare, sous les traits de Séraphin Poudrier par exemple, n'accumule des richesses qu'en vue de retenir (non pas de s'accomplir), puisqu'il se conçoit en permanence selon un mode d'existence de non-disjonction, de potentialisation, face à la valeur recherchée. Aussi le paranoïaque, à l'instar de la victime du «horla» de Maupassant, quelle que soit sa position effective, se rêve-t-il toujours conjoint avec la valeur redoutée (en tant que sujet réalisé). Que de tels modes de jonction fictive soient de l'ordre de la présentification mise en lumière par Greimas (1986: 5-11) dans son étude de la nostalgie, ou du théâtre mental assimilé à la fidélité, chez Zilberberg (1985: 349-379), ils pointent l'irruption en discours de l'intensité affective. Le simulacre modal renvoie de cette manière aux premières esquisses tensives entourant une ombre de valeur, lesquelles assurent au sujet une homogénéité processuelle:

[l]a configuration passionnelle comprendrait [...] un principe régissant, partiellement indépendant des modalisations proprement dites [, qui] se manifesterait sous la forme d'une aspectualisation et renverrait, au niveau des modulations tensives, à un «style sémiotique» spécifique.

(Greimas et Fontanille, 1991: 67-68)

Ainsi, dans Bonheur d'occasion, le simulacre modal que Florentine Lacasse entretient à l'égard de Jean Lévesque illustre clairement la syntaxe transitoire et cursive du substrat passionnel, gérée par une image but. La position de non-conjonction de Florentine (par rapport à l'homme aimé qui l'abandonne) est productrice d'un faisceau complexe d'affects imbriqués, portés par une aspectualisation itérative, qui trahissent sa détresse. Se manifestant au moment de son trajet vers l'ancien domicile de Jean, ceux-ci traduisent d'abord de la peur :

Elle ne s'avouait pas encore que toutes ses démarches pour retrouver Jean demeuraient vaines. Accablée par son malheur, éperdue de crainte, elle s'imagina que le hasard la servirait ce jour même [...]. (Roy, 1993: 258)

La situation de non-conjonction nourrit ensuite un sentiment de solitude: "Alors Florentine s'aperçut qu'elle était seule au monde avec sa peur. Elle entrevit la solitude, non seulement la solitude à elle, mais la solitude qui guette tout être vivant» (ibid.: 261). Survient alors un sentiment de rancune : «Et au fond 
de son être, elle découvrit aussi, impitoyable, clair, un affreux sentiment de rancune, quelque chose de si laid qui poussait là qu'elle en fut comme empoisonnée» (ibid.: 262). La rancune se transforme en humiliation:

Il aimerait d'autres femmes. Il lui faudrait un effort de mémoire peutêtre pour parvenir à l'évoquer, elle, Florentine. [...] Mais tant qu'il vivrait, tant qu'il respirerait, elle connaîtrait l'humiliation de n'avoir pas su le retenir. (Ibid.)

La séquence passionnelle se clôt par un état affectif de vengeance qui ne se résorbe pourtant pas:

Aussitôt, elle résolut d'acheter le petit chapeau convoité [...].

Ainsi elle se vengerait de Jean. [...] Elle serait si élégante que, s'il

la rencontrait un jour par hasard, il regretterait de l'avoir

délaissée. Mais ce serait à son tour à elle de se montrer impitoyable. (Ibid. : 266)

On le voit, ce type de mise en commun des modes d'existence et des simulacres existentiels à corrélats phoriques et aspectuels produit une charpente autodynamique ${ }^{18}$, où le sujet passionnel devient une structure du mixte, sous l'emprise des arrangements, des intersections et des combinaisons d'ordre modal (Fontanille et Zilberberg, 1998: 193).

Le chemin qui mène de Du sens à Sémiotique des passions invite à apprécier l'enrichissement remarquable de la problématique des modalités. Celles-ci peuvent être traitées soit comme des grandeurs simples et discrètes (discontinues), ce qui permet de les assimiler à la compétence des sujets narratifs, soit comme des grandeurs tensives et intermodales (continues), les renvoyant à la composition des dispositifs passionnels. Dans le second cas, ce sont des valeurs modales, obéissant en tout point à la définition tensive des valeurs en général: «leur "valeur" est conditionnée par une corrélation, converse ou inverse, entre leur intensité et leur extensité" phorique (ibid.: 232). Alors que la mise en discours des modalités associées aux «épreuves qualifiantes» du schéma narratif se trouve régie par les étapes de la conversion et sa prise en compte par les procédures de l'énonciation, les modalités participant aux dispositifs passionnels relèvent du travail de la praxis énonciative. Véritable opérateur pathémique, le sujet de l'énonciation assure la sensibilisation de la syntaxe intermodale en intégrant, lors de la mise en discours, les grandeurs tensives aux valeurs modales. De plus, il projette sur divers axes syntaxiques, soit sur les sujets, soit sur les objets, soit sur la jonction, un style passionnel redevable à la tensivité originaire. Loin donc d'être la propriété exclusive du sujet anthropomorphe (narratif), les effets passionnels relèvent du discours tout entier, ce qui implique qu'ils requièrent, pour se manifester, l'intervention du sujet énonciateur dont on parvient à retracer les contours pathémiques grâce à ses opérations constitutives: le sujet se définit au fond comme un effet de ses «convocations"(Bertrand, 2000: 58). Henri Quéré disait, de la théorie sémiotique, que la consolidation de ses acquis «devait laisser place, à côté des points aveugles et des manques à combler, à la possibilité de développements encore insoupçonnés» (1993: 283 284). Au vu de l'auscultation du sens, on constate que le trajet parcouru par la théorie répond justement à une telle visée. On pourrait en effet reprendre en un tableau synthétique les stations méthodologiques complémentaires qui qualifient aujourd'hui l'essentiel de l'œuvre greimassienne:

Les liens entre modulations et modalités, entre aspect et procès, entre sensations d'ordre proprioceptif associé au thymisme et à la physiologie de l'affect, d'une part, et les catégories intéroceptive et extéroceptive de la cognition, de la perception, et de l'action dans le monde de l'autre [...] sont exemplaires des rapports que [Greimas et Fontanille] établissent entre la théorie standard et la nouvelle sémiotique.

(Broden, 1995: 235-236; nous traduisons) ${ }^{19}$

\section{POUR CONCLURE}

Que l'on ne se méprenne pas: la fortune de $D u$ sens échappera difficilement au débat, compte tenu de l'investissement de plus en plus marqué de la sémiotique par une épistémologie continuiste axée sur la praxis énonciative. Dans un hommage à l'œuvre de Greimas largement centré sur l'article programme que constitue "Éléments d'une grammaire narrative» (DS: 157-183), Jacques Geninasca rappelle les failles dont 
on a taxé le fameux principe de conversion, notamment en ce qui a trait à la mise en corrélation des opérations constitutives de l'axe des contradictoires (ou «schéma») et des énoncés de faire impliqués par la "polémique» (1997: 52). Si, d'après le chercheur, le parcours génératif tout comme le schéma narratif canonique se sont avérés, avec le temps, dépourvus de valeur opératoire, les réajustements à la fois épistémologiques et théoriques de la sémiotique, favorisés par l'élaboration d'une théorie des passions, situent aujourd'hui le couple conversion-convocation du côté des opérations énonciatives du discours. Bénéficiant des découvertes (sinon de leurs accidents) issues d'une prise en compte de plus en plus raffinée des dimensions perceptive, sensorielle et affective des textes, de même que d'une conceptualisation du discours en acte, la théorie se définit en fonction d'un nouvel objet:

L'objet de la théorie, ce ne sont pas tant les représentations sémantiques qui seraient en amont des discours, mais les conditions auxquelles doit satisfaire un objet à vocation sémiotique pour qu'on puisse le construire comme texte satisfaisant à tel ou tel ensemble de conditions de cohérence.

Geninasca poursuit en soulignant qu'au lieu de postuler l'existence d'une structure $a b$ quo dont dépendrait le sens du discours, il convient d'étudier ce vers quoi s'est dirigée la sémiotique des passions les conditions de cohérence des énoncés partiels ou globaux et de reconnaitre que la production/saisie des discours est indissociable de la production/saisie d'un texte, dont l'objet textuel demeure la trace virtuelle.
Dans ce contexte nouveau, on substituera la question des mécanismes de conversion dont on n'a jamais proposé [...] une grammaire, la problématique du concours des structures qui conditionnent l'ensemble complexe des opérations dont dépend le sens. (Ibid.)

La postérité montrera, en effet, que c'est en vertu de son souci de cohérence que Greimas est demeuré fidèle à son entreprise; que cette notion s'applique à la générativité du sens ou à la saisie des invariants narratifs, dont témoigne $\mathrm{Du}$ sens, ou à la volonté de circonscrire le rôle de la proprioceptivité corporelle, dans notre accès à l'univers du sens, illustrée par Sémiotique des passions. À l'avis de Pierre Ouellet, [...] on ne peut plus parler Du sens, comme Greimas, jadis, dans le titre de deux de ses principaux ouvrages, sans parler $d u$ même coup Des sens, comme Greimas lui-même le fait dans sa dernière œeuvre publiée, De l'imperfection. (1997: 8)

On est ainsi tenu de reconnaître que l'approche résolument "constructiviste» (Landowski, 1997: 253) du maître a permis à la théorie sémiotique de rendre compte des procès de composition et de négociation à l'œuvre, que ce soit sur le plan de ses reconstructions méta-discursives, ou sur le plan des échanges discursifs au niveau de l'analyse. En clair, la quête de la cohérence qui transcende l'ensemble de l'œuvre théorique de Greimas réaffirme l'ampleur programmatique de la démarche adoptée, dont ressort surtout une vigueur incontestable. 


\section{N O TES}

1. Dorénavant, tous les renvois à cet ouvrage se feront à partir de cette édition à l'aide du sigle DS, suivi de la pagination, et mis entre parenthèses dans le corps du texte.

2. A. Hénault précise que l'acte épistémique, décrit par les termes affirmer, refuser, admettre, douter, "ne passe pas par des contradictions, des étapes tranchées, distinctives, il saisit des moments se métamorphosant par des plus ou des moins" (1992a: 117).

3. P. Fabbri signale: "C'est à l'esthétique qu'il appartient de renouer le pacte originel entre le sensible et le sujet sentant, d'où affleure, au fil de la perception, la pensée. Le sentir n'a pas de privilège esthétique, mais c'est à son niveau que s'interroge l'unité “trans-sensible” avant la diversification des sens. Dans le sentir "primitif" se maintient et se module la riche totalité symbiotique et synergétique des sens [...]» (1992: 23).

4. On repère ces tendances, à titre d'exemple, en physique quantique, en thermodynamique dans le concept d'énergie et en biologie dans la notion de probabilités statistiques. Prigogine est un des principaux vulgarisateurs de ces tendances propres à l'épistémologie scientifique. 5. C. Zilberberg (1992a: 10, note 13) souligne que cette réflexion fondée sur la notion de "même», à partir de laquelle s'engendrent les valeurs, se trouve au cœur de l'épistémologie structurale. Chez Hjelmslev, l'engendrement des fonctifs se fait à partir de la fonction; chez Greimas, l'engendrement des contraires se produit à partir de tel axe sémantique.

6. La dimension thymique du sens (être) est complémentaire aux dimensions cognitive (savoir) et pragmatique (faire) du discours. Du grec thumos, le thymisme (euphorie/dysphorie) désigne le cœur, notion réarticulée par la théorie en termes de phorie, soit le sentir qui engage les réactions corporelles. Cette catégorie primitive s'avère être inséparable du corps sensible; elle permet de décrire la manière dont «tout être vivant s'inscrit dans un milieu, "se sent" lui-même et réagit à son environnement" (Greimas, 1983: 93). Précisément parce qu'elle se trouve le point médiateur entre l'extéroceptivité (se rapportant aux sensations et partant aux figures du monde) et l'intéroceptivité (décrivant notre perception du monde intérieur sous forme de concepts, d'impressions, de souvenirs et de sentiments), la proprioceptivité, porteuse de "protensivité» liant le sujet à son objet, constitue un terme à la fois neutre et complexe: le monde et la conscience n'existent, en tant qu'univers de significations, que par cette frontière irréductible du corps sensible où se conjuguent en une même sensation l'expérience de soi et l'expérience du monde.

7. Voir à ce sujet, notamment "De la modalisation de l'être" (Greimas, 1983: 93-102).

8. À Greimas de préciser: «Si la modalité du vouloir valorise l'objet, cet objet, en tant qu'actant de l'énoncé modal, peut être converti soit en un énoncé descriptif $d u$ faire [Jean veut que Pierre parte/Pierre veut partir] - et le faire en tant que tel se trouve valorisé - soit en énoncés attributifs [Pierre veut une pomme/Pierre veut être bon] - et l'actualisation du vouloir s'exprime alors par la possession d'objetsvaleurs indiqués dans les énoncés attributifs» (DS: 171).

9. Sur l'exclusion du Destinateur, la théorie des prégnances, bien qu'elle n'aborde pas la notion de protensivité de la même manière, rejoint la théorie des passions: «en-deçà [...] de ce procès cognitif modalisant la relation intentionnelle de "désir" Sujet-Objet, il y a un rapport d'affection thymique et de vouloir (d'appétition) liant le sujet à des prégnances figurativisées, un rapport de nature fondamentalement esthétique que nous appellerons affect esthétique» (Petitot, 1985: 295; l'auteur souligne).

10. H. Parret s'inspire de la Critique du jugement d'Emmanuel Kant. 11. Les modalités de la passion se rapportent à la position existentielle (attitude, sentiment, croyance, etc.) du sujet face à la valeur recherchée. 12. A. J. Greimas et J. Courtés rappellent que «[l]'extensivité des structures modales est [...] une composante essentielle de l'identité de l'acteur, c'est-à-dire de sa permanence en discours " (1979:178-179). Malgré la diversité de points de vue adoptés, la question des modalités représente un des domaines de recherche les plus féconds en sémiotique greimassienne; voir J. Fontanille et C. Zilberberg (1998: 169-196).

13. Dictée par le vouloir, la valeur pour le sujet débouche sur une structure modale: $V=m e(s)$, où " $\mathrm{s}$ " désigne une grandeur sémique quelconque, sélectionnée lors de la conversion, et «me " renvoie à une des modalités sélectionnées, le «e " désignant la relation existentielle modifiée par la modalisation (Greimas, 1983: 100).

14. Délié du modèle proppien et ayant pour unique appui la syntaxe modale, le schéma narratif canonique devient justiciable de généralisation et se prête à l'analyse des discours qui débordent le conte ou d'autres genres anthropomorphes, à savoir le discours en sciences sociales. Pour une synthèse de ces développements, voir Greimas (1989: 524-544).

15. Pour Greimas et Fontanille, «toute relation objectale recouvrirait une intersubjectivité potentielle [...]» (1991: 61). Ce postulat n'est pas sans rappeler la description des structures polémico-contractuelles inhérentes à la colère: "[L]e couple de héros et de traître, de sujet et d'anti-sujet, n'est pas le résultat d'une articulation catégorielle binaire, mais d'une présupposition réciproque qui les rend inséparables, l'un n'apparaissant jamais sans la présence concomitante de l'autre" (Greimas, 1983: 243).

16. Ils précisent: «[L]e simulacre est une configuration qui résulte [...] de l'ouverture d'un espace imaginaire par l'effet des charges modales qui affectent le sujet: [...] les changements "imaginaires" des rôles actantiels, c'est-à-dire tout ce qui affecte la représentation syntaxique des énoncés de jonction, sont les principales propriétés de ces simulacres [...]»(Greimas et Fontanille, 1991: 63).

17. J. Fontanille (1986) passe en revue des solutions proposées à la problématique de l'intermodalité, mais incomplètes, à savoir les "confrontations modales" formulées par A. J. Greimas, les «suites modales » de J.-C. Coquet ainsi que les «concaténations modales " instituées par H. Parret.

18. D. Bertrand précise: "Combinées avec les catégories modales, les catégories aspectuelles permettent de déterminer avec une précision accrue le "mode d'existence" des objets sémiotiques : virtualisation, actualisation, réalisation. Cette problématique [...], ancrée dans la tradition philosophique et linguistique, est [...] particulièrement cruciale pour l'analyse des processus émotionnels et passionnels, caractérisés par la production des simulacres et la "gestion" de leurs modes de présence» $(1987: 2)$.

19. "The links between modulations and modalities, between aspect and process, between proprioceptive sensations associated with thymia and the physiology of affectivity, on the one hand, and the interoceptive and exteroceptive categories of cognition, perception, and action in the world on the other [...] exemplify the connections GF asserts between the standard theory and the new semiotics". 


\section{RÉFÉREN CES BIBLIO G RAPH IQ U ES}

BERTRAND, D. [1984] : «Narrativité et discursivité : points de repère et problématiques", Actes sémiotiques. Documents, vol. VI, n 59, 1-38;

[1987]: «Le corps émouvant. L'absence. Propositions pour une sémiotique de l'émotion ", Versus, nos 47-48, 1-13;

— [2000]: Précis de sémiotique littéraire, Paris, Éd. Nathan, série

"Linguistique".

BRANDT, P. A. [1986] : Entrée "passion ", dans A. J. Greimas et

J. Courtés (1986), 163-165

Broden, T. F. [1995]: «Commemorative Essay: A. J. Greimas (1917-

1992)", Semiotica, vol. 105, nos 3-4, 207-242.

COllot, M. [1997]: La Matière émotion, Paris, PUF.

FABBRI, P. [1992]: «Échappée», Nouveaux Actes Sémiotiques, no 19

("Pertinence et adéquation»), 19-30.

FONTANILLE, J. [1986]: «Le tumulte modal. De la macro-syntaxe à la micro-syntaxe passionnelle», Actes sémiotiques. Bulletin, vol. IX, no 39 ("Les passions. Explorations sémiotiques»), 12-31.

FontANille, J. et C. Zilberberg [1998]: Tension et Signification, Liège, Mardaga.

GENINASCA, J. [1997] : «Et maintenant?», dans E. Landowski (1997),

41-57.

GreimAS, A. J. [1966] : Sémantique structurale. Recherche de méthode, Paris, Larousse, coll."Langue et langage»;

[1970]: Du sens. Essais sémiotiques, Paris, Seuil;

_ [1983]: Du sens II. Essais sémiotiques, Paris, Seuil;

_ [1986] : «De la nostalgie», Actes sémiotiques. Bulletin, vol. IX, no 39

("Les passions. Explorations sémiotiques»), 5-11;

- [1987]: De l'imperfection, Périgueux, Fanlac;

[1989]: "On Meaning", New Literary History, vol. 20, no 3

("Greimassian Semiotics»), printemps, 539-550.

Greimas, A. J. et J. COURTÉS [1979]: Sémiotique. Dictionnaire raisonné de la théorie du langage, tome I, Paris, Hachette;

- [1986]: Sémiotique. Dictionnaire raisonné de la théorie du langage.

Compléments, débats et propositions, tome II, Paris, Hachette.

Greimas, A. J. et J, FonTANILle [1991]: Sémiotique des passions. Des états de choses aux états d'âme, Paris, Seuil.

HÉNAUlT, A. [1992a]: Histoire de la sémiotique, Paris, PUF, coll. «Que

sais-je?", n०2691;

[1992b]: «Compte rendu de Sémiotique des passions de A.J.

Greimas et Jacques Fontanille», RS-SI, vol. 12, n 3, 185-196.

KLINKEnBERG, J.-M. [2002]: Entrée "sémiotique», dans P. Aron,

D. Saint-Jacques et A. Viala (dir.), Le Dictionnaire du littéraire, Paris,

PUF, 547-548.

LANDOWSKI, E. [1997]: «Le sémioticien et son double», dans
E. Landowski (dir.), Lire Greimas, Limoges, Presses universitaires de Limoges, 229-255.

LATELlA, G. [1986] : Entrée "sujet», dans A. J. Greimas et J. Courtés, tome II, 214-215.

Merleau-PonTy, M. [(1945) 1976] : La Phénoménologie de la perception, Paris, Gallimard.

OUellet, P. [1992]: Voir et Savoir. La perception des univers du discours, Candiac, Éd. Balzac, coll. "L'Univers des discours"; (dir.) [1997]: Action, passion, cognition d'après A.J. Greimas,

Québec et Limoges, Nuit Blanche éditeur et Presses universitaires de Limoges;

- [2000]: Poétique du regard. Littérature, perception, identité, Québec et Limoges, Éd. du Septentrion et Presses universitaires de Limoges. PARret, H. [1986]: Les Passions. Essai sur la mise en discours de la subjectivité, Bruxelles, Mardaga.

PARRET, H. et H.-G. RuPRECHT (dir.) [1985] : Exigences et perspectives de la sémiotique. Recueil d'hommages pour Algirdas Julien Greimas, tomes 1 et 2, New York, John Benjamins Publishing Company.

PETITOT, J. [1985]: «Les deux indicibles, ou la sémiotique face à

l'imaginaire comme chair ", dans H. Parret et H.-G. Ruprecht (dir.), tome $1,283-305$.

PozzATO, M. P. [1991]: "Le monde textuel», Nouveaux Actes Sémiotiques, $\mathrm{n}^{\circ} 18$.

QUÉRÉ, H. [1993] : «Compte rendu de A. J. Greimas et Jacques Fontanille, Sémiotique des passions. Des états de choses aux états d'âme", Zeitschrift für Französische Sprache und Literatur, CIII/3, 283-285. RICEUR, P. [1985]: "Figuration et configuration, à propos du Maupassant de Greimas ", dans H. Parret et H.-G. Ruprecht (dir.), tome 2, 801-809

— [1993]: "Partout où il y a signe... ", Nouveaux Actes Sémiotiques, n 25 ("Hommages à A. J. Greimas"), 45-48.

RoY, G. [(1945) 1993]: Bonheur d'occasion, Montréal, Éd. du Boréal; - [1954]: Alexandre Chenevert, Montréal, Beauchemin.

ZilberberG, C. [1985] : "Conversion et réversion ", dans H. Parret et H.-G. Ruprecht (dir.), tome 1, 349-379 $\mathrm{n}^{\mathrm{os}} 23-24$; [1992a]: "Présence de Wölfflin", Nouveaux Actes Sémiotiques, _ [1992b]: «Défense et illustration de l'intensité », dans J. Fontanille (dir.), La Quantité et ses modulations qualitatives, Actes du colloque "Linguistique et Sémiotique II ", Limoges et Amsterdam, Presses universitaires de Limoges et Benjamins, 75-109;

_ [1993]: «Le schéma narratif à l'épreuve», Protée, vol. 21, no 1 ("Schémas»), hiver, 65-87. 